\title{
Relativistic computation
}

\author{
Andréka, H., Madarász, J., Németi, I., Németi, P. and Székely, G.
}

June 1, 2017

\section{Introduction}

Two major new paradigms of computing arising from new physics are quantum computing and general relativistic computing. Quantum computing challenges complexity barriers in computability, while general relativistic computing challenges the physical Church-Turing thesis itself. In this chapter, we concentrate on relativistic computers and on their challenge to the physical Church-Turing thesis (PhCT).

The PhCT concerns the belief that whatever physical computing device (in the broader sense) or physical thought experiment that will be designed by any future civilization, it will always be simulateable by a Turing machine, in some sense. In a somewhat more concrete form, the PhCT says that for any function $f: N \rightarrow N$, where $N$ is the set of natural numbers, if $f$ is physically realizable then it is also Turing computable. It is worthwhile to make explicit what we mean by "physically realizable". Roughly, $f$ is physically realizable if there is a physical thought experiment in which $f$ is computed according to the present laws of nature. By a "physical thought experiment" we mean the following. We specify a recipe or design for a possibly futuristic experiment, and show that it is consistent with the presently known laws of physics that if our far future descendants were to perform this experiment, the outcome for input $n \in N$ would be $f(n)$. The design we present in the present chapter is an arrangement in the physical world that operates on finite data in accordance with a finite program. Hence it is a challenge to the physical Church-Turing thesis as formulated in [10]: "Whatever can be calculated by a machine (working on finite data in accordance with a finite program of instructions) is Turing machine computable." More detail is given in [17, 
p.351, around Thesis 2']. Piccinini [34] calls this thesis the Modest Physical Church-Turing Thesis. ${ }^{1}$

Intuitively, a Turing machine is a finite-state automaton, TM, which has access to a finite but indefinitely extendible tape. TM can add a cell to the tape, it can write on a cell of the tape, and it can read a cell of the tape depending on its state. TM can also move along the tape. A typically nonTuring-computable task is one which needs to survey the result of a given algorithm for all natural numbers $n$ before being able to return an answer. Such tasks include deciding recursively enumerable sets. In this chapter, we design a physical computer for a thought experiment operating in general relativistic spacetimes, which can decide any recursively enumerable set. Thus, it can compute a non-Turing-computable task. Our relativistic computer is a team consisting of a computer, a Turing machine, and a programmer all operating in the general relativistic spacetime of a slowly rotating black hole. ${ }^{2}$ The computer may work for infinite time, and the programmer can extract the desired result of its infinite work in a finite time. We investigate whether this physical computer could be realized by a future civilization.

A positive contribution of this chapter is that we answer some worries published in $[34,12,29]$. Namely, we address the questions whether the probability of error should be 1 for a machine working for infinite time, whether the transfer of results between the computer and the programmer is possible, and whether spacetimes allowing the operation of such a relativistic computer are physically realistic.

The layout of the present chapter is as follows. In section 2, we outline the idea of a relativistic computer. In section 3, we define what an abstract relativistic computer is, and we investigate what kinds of relativistic spacetimes can harbor such computers. In section 4, we deal briefly with how physically realistic two aspects of this abstract physical computer are. In section 5, we survey results about the complexity of sets of numbers that can be computed by relativistic computers. We close the chapter with a conclusion in section 6.

\footnotetext{
${ }^{1}$ Related work on transcending the physical Church-Turing thesis using designs based on physical considerations can be found, e.g., in $[6,8,24,40,41]$.

${ }^{2}$ There are many other general relativistic spacetimes, besides the one of the slowly rotating black hole, in which such an arrangement can be done, see section 3 .
} 


\section{Design for a relativistic computer}

This chapter serves as a motivation for the next, more formal, one. We will use intuitive language, similar to the one in Rindler's relativity book [38]. ${ }^{3}$ In the next section, we will define formally all the notions we use.

\subsection{A fairy tale}

Imagine a computer $\mathrm{C}$ which can compute arbitrarily fast. More precisely, the programmer $\mathrm{P}$ can speed up $\mathrm{C}$ with an arbitrary (but finite) rate. For example, in the first 10 minutes of its operation $\mathrm{C}$ may execute 10 steps. Then in the next 5 minutes it executes another 10 steps. After this, in the next 2.5 minutes it executes a third 10 steps. And so on. So, in the first $20=10+5+2.5+\ldots$ minutes of its operation, $\mathrm{C}$ performs infinitely many steps. Hence, in the first 20 minutes, $\mathrm{C}$ performs the same number of computational steps as an ordinary Turing machine would do in the whole of its infinite lifetime. Such a $\mathrm{C}$ could be used to decide an uncomputable problem, e.g., it could decide the Halting Problem of Turing machines, or the consistency of ZF set theory, or it could decide any recursively enumerable set of numbers. Clearly, C could be a "super-computer": it could outperform ordinary Turing machines, in particular it could challenge the $\mathrm{PhCT}{ }^{4}$

Unfortunately, according to present-day physics, physically realizing this $\mathrm{C}$ is not possible. To alleviate this problem, in the present work we will gradually refine the design of $\mathrm{C}$ such that it will approach being physically possible.

\subsection{Relativity theory}

In the above situation, a hope is offered by relativity theory, as relativity theory makes it possible to manipulate time. It can be arranged, by taking suitable journeys, that for some observers, time runs faster while for others, time runs slower. This is usually visualized by saying that wristwatches, or simply clocks, of the first observer run faster while clocks of the other run

\footnotetext{
${ }^{3}$ The final stage of the intuitive plan for creating an infinite speed-up effect we outline in this section is elaborated in concrete mathematical detail in [17].

${ }^{4}$ Copeland [9] calls this computer an Accelerating Turing Machine. Other names used are Zeno machine [2], Zeus machine [40], and Plato machine [12].
} 
slower. Of course, this does not mean the speeding up of only clocks, but of all atomic and sub-atomic processes. ${ }^{5}$

Consider first the simpler case of special relativity. The Time Dilation theorem of special relativity states that clocks of a fast moving observer run slower, as measured by a stationary observer. ${ }^{6}$ As the velocity of the observer approaches the speed of light, his clocks gradually freeze. Now, in our design for a relativistic computer, instead of making the clocks of the computer run faster, we make the clocks of the programmer run slower. Let us put the programmer in a spaceship and put the computer on the Earth. If the spaceship moves fast enough, its clocks will run arbitrarily slow, hence the clocks on the Earth will run arbitrarily fast relative to the clocks of the programmer in the spaceship. The programmer can accelerate his spaceship in such a manner that finite time will pass for him while the computer computes for an infinite time on Earth (according to Earth-clocks). However, for achieving this, the programmer has to endure unbounded acceleration, which according to present-day knowledge, will kill him. Because of this and because of other problems we would face following this setting ${ }^{7}$, we abandon special relativity and we refine our design for a relativistic computer by turning to general relativity.

\subsection{Gravitational time--dilation}

If we switch from special relativity to general relativity, the problem of unbounded acceleration may be overcome by "letting general relativistic spacetime do the work of distorting time" (instead of using solely the programmer's engine for this purpose).

Let us start out from the so-called Gravitational Time Dilation effect (GTD). The GTD is a theorem of relativity theory: it says, sloppily, that

\footnotetext{
${ }^{5}$ In the next section, wristwatch time will be called proper time along worldlines.

${ }^{6}$ This is connected to the popular theorem known as Twin Paradox of relativity: if one of two twins takes a journey in which he travels fast, he will be younger than his twin at the time or reunion. For an experimental confirmation, see [5].

${ }^{7}$ Pitowsky [36, sec.2] designs a spaceship circling the Earth faster and faster so that finite time passes for him while the computer on the Earth has an infinite time to compute. Assuming that the programmer can endure the unbounded acceleration, he will learn if $\mathrm{ZF}$ is inconsistent since the proof of inconsistency will be found at a concrete time and sent to him. However, he will never be in a situation to know that ZF is consistent if it is so, since in his life there is no event "after" the computer's infinite time. In technical terms, special relativistic spacetime is not a Malament-Hogarth one: see section 3.
} 
gravity makes time run slow. More sloppily: gravity slows time down. Clocks that are deep within gravitational "wells" run slower than ones that are farther out. We will have to explain what this means, but before explaining it we mention that GTD is not only a theorem of general relativity. This theorem, GTD, can be proved already in special relativity in such a way that we simulate gravity by acceleration; see [25]. (We transfer the result from special relativity to general relativity by using Einstein's Equivalence Principle which states the equivalence of inertial and gravitational mass, see, e.g., [38, p.18].) So one advantage of GTD is that why it is actually true can be traced down by using only the simple methods of special relativity. Another advantage of GTD is that it has been tested several times, and these experiments are well known. Actually, the General Positioning System (GPS) of today's technology tests GTD on a daily basis: each time GPS is used, say for locating a car, GTD is tested [37].

Roughly, GTD can be interpreted by the following experiment. Choose a high enough tower on the Earth, put precise enough (say, atomic) clocks at the bottom of the tower and the top of the tower, then wait enough time, and compare the readings of the two clocks. Then the clock on the top will run faster (show more elapsed time) than the one in the basement, at each time one carries out this experiment. 8 Gravity "causes" the clock on the top ticking faster than the one in the basement. Therefore computers there also compute faster, if only slightly. How could we use GTD for designing computers that compute more than Turing machines can? In the above outlined situation, by using the gravity of the Earth, it is difficult to make practical use of GTD. However, instead of the Earth, we could choose a huge black hole.

\subsection{Ordinary black hole}

A black hole is a region of spacetime with such a strong "gravitational pull" that even light cannot escape from this region. There are several types of black holes. ${ }^{9}$ We begin to demonstrate the main ideas of a relativistic computer in the case of the simplest, spherically symmetric black hole, called a Schwarzschild spacetime.

\footnotetext{
${ }^{8}$ Actually, this experiment was done at Harvard University's Jefferson tower in 1960 by Pound and Rebka [38, p.28].

${ }^{9} \mathrm{~A}$ good source is Taylor and Wheeler [42] or Thorne [43].
} 
As we move closer and closer to the black hole, the gravitational pull gets stronger and stronger. By this we mean that it is harder and harder to maintain distance from the black hole. Actually, there is a distance where the gravitational pull is so strong that we cannot maintain altitude any more and we are pulled toward the black hole inevitably. These "no-return" places form a sphere around the center of the black hole which is called the "eventhorizon".

Let us study observers suspended over the event horizon. "Suspended" means that the distance between the observer and the event horizon does not change. This "suspending" of the observers can be done, for example, by letting their spaceships hover over the event horizon, using their rockets for maintaining altitude. (This is rather similar to ourselves being suspended over the Earth, where, instead of rockets, the electromagnetic forces in the crust of the Earth keep us "suspended".) As we approach the event horizon from far away outside the black hole, the gravitational "pull" of the black hole approaches infinity as we get closer and closer to the event horizon. We can measure this gravitational pull as the acceleration of "apples" let go by a suspended observer, or equivalently, by the amount of fuel needed to maintain height of a suspended observer.

Assume one suspended observer $\mathrm{C}$ (for computer) is higher up and another one, $\mathrm{P}$ (for programmer), is suspended lower down. So, $\mathrm{C}$ sees $\mathrm{P}$ below her while $\mathrm{P}$ sees $\mathrm{C}$ above him. Now the GTD will cause the clocks of $\mathrm{P}$ to run slower than those of $\mathrm{C}$, that is, the clocks of $\mathrm{C}$ will run faster than those of P. Moreover, they both agree on this if they are watching each other, e.g., via photons (light-signals). Let us keep the height of $\mathrm{C}$ fixed. If we gently lower $\mathrm{P}$ towards the event horizon, the ratio between the speeds of their clocks increases. Moreover, as $\mathrm{P}$ approaches the event horizon, this ratio approaches infinity. This means that for any integer $n$, if we want C's clocks to run $n$ times as fast as P's clocks, then this can be achieved by lowering $\mathrm{P}$ to the right position. To achieve an "infinite speed-up" we could do the following. We could lower P towards the event horizon such that P's clocks slow down (more and more, beyond limit) in such a way that there is a certain finite time-bound, say $b$, such that, roughly, throughout the whole history of the universe, $\mathrm{P}$ 's clocks show a time smaller than $b$. More precisely, by this we mean that whenever $\mathrm{C}$ decides to send a photon to $\mathrm{P}$, then $\mathrm{P}$ will receive this photon before time $b$ according to P's clocks. We can do this as follows, for example. After 1 hour has passed in C's life, P lowers to a height where, suspended, his time is twice as slow as C's one, so while 1 more hour passes in 
C's life, in P's life only $1 / 2$ hour passes. Then $\mathrm{P}$ goes even lower such that in his life only $1 / 4$ hour passes while in C's life 1 hour passes. And so on. Apart from the times needed for lowering, then in P's life $2=1+1 / 2+1 / 4+\ldots$ hours pass while in C's life an infinite time passes.

However, as $\mathrm{P}$ gets closer and closer to the event horizon, the gravitational pull tends to infinity and this will kill him. This is analogous to the situation in special relativity where the unbounded acceleration kills our programmer P. If $\mathrm{P}$ does not approach the event horizon through stages of being suspended, but instead he falls into the black hole without using rockets to slow his fall, then he does not have to withstand the gravitational pull of the black hole. He would only feel the so-called tidal forces which can be made negligibly small by choosing a large enough black hole. However, his falling through the event horizon would be so fast that some photons sent after him by $\mathrm{C}$ would not reach him outside the event horizon. Thus $\mathrm{P}$ has to approach the event horizon relatively slowly in order that he be able to receive all possible photons sent to him by C. In theory, he could use rockets for this purpose, i.e. to slow his fall. However, we cannot compromise between the two requirements by choosing a well-balanced route for P: no matter how he would choose his route, either $\mathrm{P}$ will be crashed by the gravitational pull, or some photons sent by $\mathrm{C}$ would not reach him. This is the reason why we can not base our relativistic computer on Schwarzschild black hole. ${ }^{10}$

\subsection{Rotating black hole}

To solve this problem, we would like to slow down the "fall" of P not by brute force (e.g. by rockets), but by an effect coming from the structure of spacetime itself.

We use a huge, slowly rotating black hole, called slow-Kerr in the physics literature [32]. In our slowly rotating black hole, besides the gravitational pull of the black hole there is a counteractive repelling effect coming from the rotation of the black hole. This repelling effect is analogous to "centrifugal force" in Newtonian mechanics and will cause P's descent to slow down at the required rate. So the idea is that instead of using the rockets of $\mathrm{P}$ for slowing his fall, we use this second effect coming from the rotation of the black hole.

\footnotetext{
${ }^{10}$ In technical terms, Schwarzschild black holes are not Malament-Hogarth spacetimes, either: see section 3 .
} 
Slowly rotating black holes have two event horizons: these are bubblelike surfaces, one inside the other - see Figure 1, and [32, Figure 2.2]. The

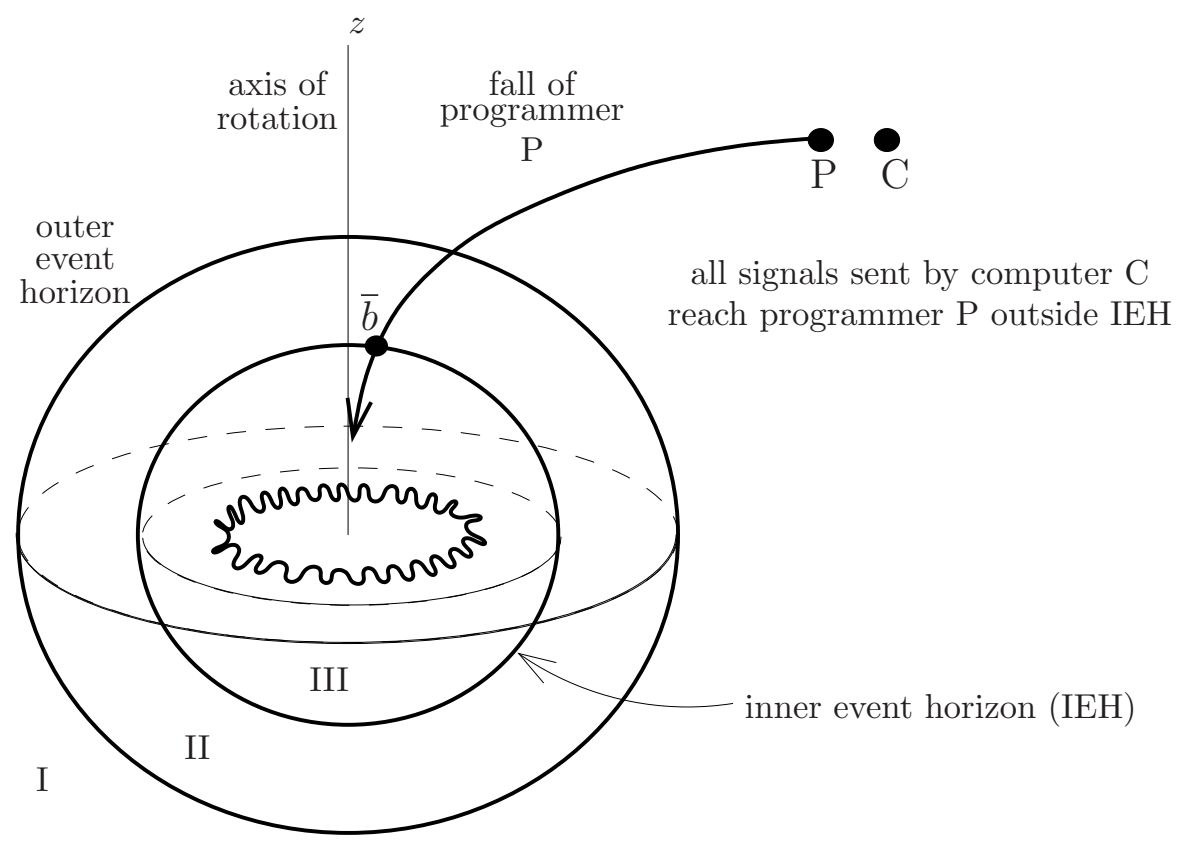

Figure 1: Cartoon-like illustration of a slowly rotating black hole. Such a black hole has two event horizons and a ring-shaped singularity. Programmer $\mathrm{P}$ can fall towards it such that he receives, before entering the inner event horizon at location $\bar{b}$, any signal the computer $\mathrm{C}$ in her infinite lifetime may send after him, and then fall inside and survive forever. The ring singularity can be avoided. The geometry of this spacetime is such that from the inner region $\mathrm{P}$ might safely escape to a new infinite universe and repeat the experiment, see section 4 and Figure 4.

outer event horizon is the result of the gravitational pull. It behaves more or less as we described in the previous subsection, and we cannot use it for our purpose. The inner event horizon marks the point where the repelling effect overcomes the gravitational effect. This is like a cushioning effect, and it makes it possible for our $\mathrm{P}$ to enter the inner event horizon in a free fall such that he can receive, before entering the inner event horizon, all the signals $\mathrm{C}$ may send after him in her infinite life. ${ }^{11}$ By this we achieved the infinite

\footnotetext{
${ }^{11}$ Details of a possible route is elaborated in [17].
} 


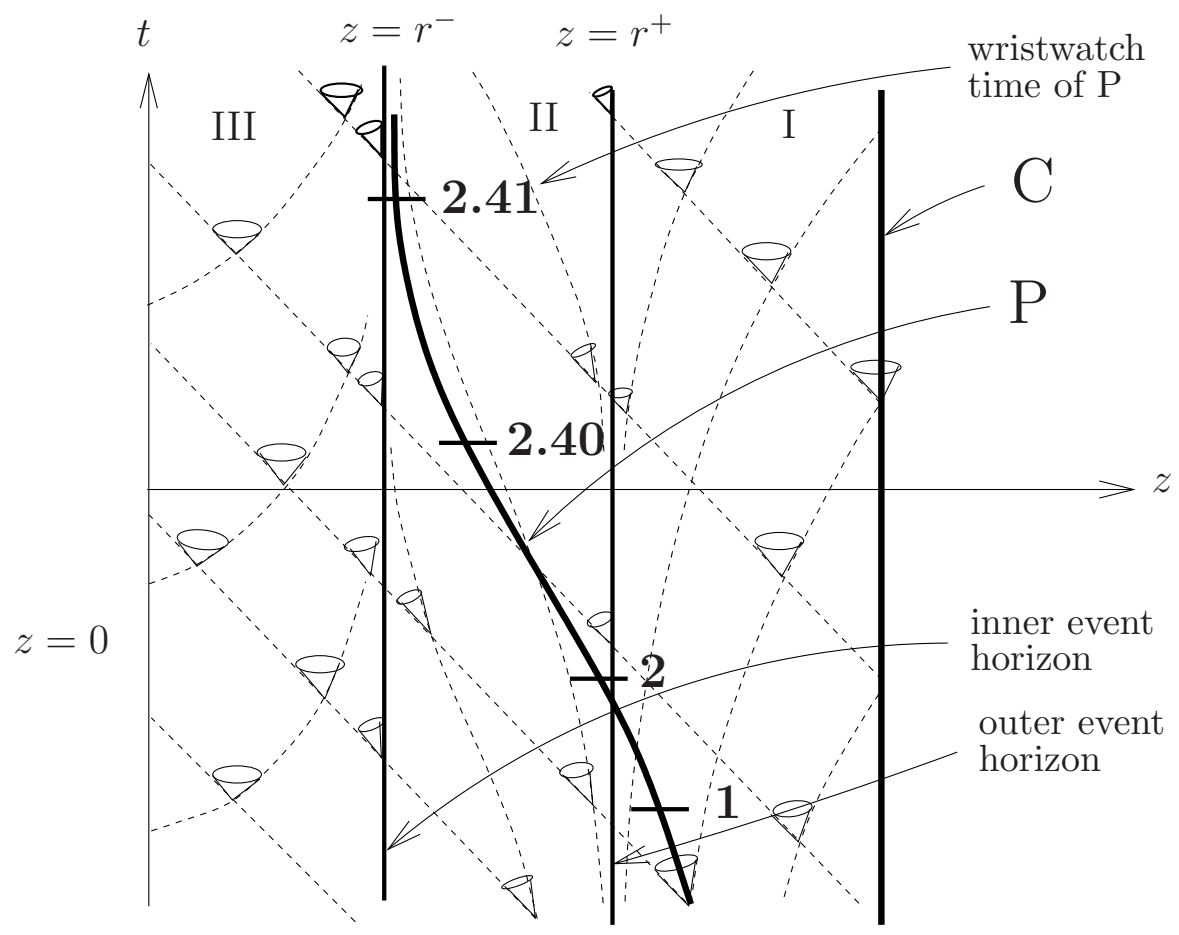

Figure 2: The "tz-slice" of the spacetime of a slowly rotating black hole in advanced Eddington-Finkelstein coordinates, [11, p.259]. This is a spacetime diagram with $t$ as the time-axis and $z$ as the axis of rotation of the black hole. For comparison with Figure $1, r^{+}$marks the worldline of the outer event horizon, $r^{-}$marks that of the inner event horizon, and the time-axis marks that of the "center" $z=0$ of the black hole. Dashed lines represent light-like geodesics. The worldline of the programmer P has to stay within the light-cones. The pattern of light cones between the two event horizons $r^{-}$and $r^{+}$illustrates that $\mathrm{P}$ can decelerate so much in this region that he will receive outside of $r^{-}$all messages sent by $\mathrm{C}$. The tilting of the light cones indicates that not even light can escape through the two event horizons. The time measured by $\mathrm{P}$ is finite (measured between the beginning of the experiment and the event when $\mathrm{P}$ meets the inner event horizon) while the time measured by $\mathrm{C}$ is infinite. A third, different kind of illustration of $\mathrm{P}$ 's worldline is in Figure 4.

speed-up we were aiming for, as represented in Figure 2. It can be seen in the figure that whenever $\mathrm{C}$ decides to send a photon towards $\mathrm{P}$, that photon 
will reach $\mathrm{P}$ before $\mathrm{P}$ meets the inner horizon.

\subsection{Implementing a relativistic computer}

We now use the above infinite speed-up scenario to describe a computer that can compute tasks which are beyond the Turing limit. We design a relativistic computer, which, given any recursively enumerable set $S$ and number $n$ as input data, will decide whether $n$ is in $S$ or not.

For input data let us choose the task, for an example, to decide whether $\mathrm{ZF}$ set theory is consistent. That is, we want to learn whether from the axioms of set theory one can derive a formula which is false. This formula can be taken to be $x \neq x$. The recursively enumerable set $S$ is the set of theorems of ZF and the computer has to decide whether $x \neq x$ is in $S$ or not. The relativistic computer starts operating as follows: In the beginning, the programmer $\mathrm{P}$ and his computer $\mathrm{C}$ are together (on Earth), not moving relative to each other, and $\mathrm{P}$ uses a finite time-period for transferring the input data, i.e., an enumerating algorithm for the theorems of ZF together with the formula $x \neq x$ to the computer $\mathrm{C}$, as well as for how $\mathrm{C}$ should send a signal to $\mathrm{P}$ when the formula $x \neq x$ is listed as a theorem of ZF. After this, $\mathrm{P}$ boards a huge spaceship, taking all his mathematician friends (relatives, everybody who is important to him, like Noah's Ark), and chooses an appropriate route towards a huge slowly rotating black hole, entering the inner event horizon when his wristwatch shows time $b$, see Figures 1,2,4. While he is on his journey towards the black hole, the computer that remained on the Earth checks one by one the theorems of ZF set theory, and as soon as the computer finds a contradiction in set theory, i.e., a proof of the formula $x \neq x$ from the axioms of set theory, the computer sends a signal to the programmer indicating that set theory is inconsistent. If it does not find a proof for $x \neq x$, the computer sends no signal. The programmer falls into the inner event horizon of the black hole, after which he can evaluate the situation. If a signal has arrived from the direction of the computer, of an agreed color and agreed pattern, this means that the computer found an inconsistency in ZF set theory; therefore, the programmer will know that set theory is inconsistent. If the signal has not arrived, and the programmer is already inside the inner event horizon, then he will know that the computer did not find an inconsistency in set theory, did not send the signal, therefore the programmer can conclude that set theory is consistent. So he can build the rest of his mathematics on the secure knowledge of the consistency of set 
theory. We note that a beneficial aspect of a rotating black hole is that, there, the programmer $\mathrm{P}$ does not have to fall into the singularity in a finite time as in an ordinary Schwarzschild black hole. He can avoid the ring singularity, and even he can decide to escape to a new region similar to the one he has started out and repeat the whole experiment. ${ }^{12}$

How does the computer $\mathrm{C}$ experience the task of this computation? $\mathrm{C}$ will see (via photons) that the programmer $\mathrm{P}$ approaches the black hole, and as he approaches it, his wristwatch ticks slower and slower, never reaching a wristwatch time $b^{-}{ }^{13} \mathrm{C}$ will see the programmer approaching the black hole in all her infinite time. For $\mathrm{C}$, the programmer shines on the sky for eternity. The only effect of C's time passing is that this image gets dimmer and dimmer, but it will never disappear. Under this sky, $\mathrm{C}$ computes away her task consisting of potentially infinitely many steps, i.e., checking the theorems of ZF one by one, in an infinite amount of time.

How does the programmer $\mathrm{P}$ experience the task of this computing? He is traveling towards the black hole, and he only has to check whether he received a special signal from the computer or not. For this task, which consists of finitely many steps, he has a finite amount of time. What would he see would he watch his team-member, the computer? He would see the computer computing faster and faster, speeding up so that when his ( $\mathrm{P}$ 's) wristwatch time reaches $b, \mathrm{C}$ would just appear to flare up and disappear. In fact, not just would the computer compute faster and faster, the time in the whole world left behind would tick faster and faster, and would flare up and disappear with the computer. This flare-up would burn P, because it carries the energy of the photons emitted during the whole infinite life of $\mathrm{C}$, which in total is infinite. In fact, we have to design a shield (or mirror) so that only intended signals from $\mathrm{C}$ can reach $\mathrm{P}$. This means that we have to ensure that $\mathrm{P}$ does not see $\mathrm{C}$ ! P's task is to watch whether there is one special kind of signal coming through this shield. (We return to protecting $\mathrm{P}$ in section 4.1.) All in all, P's task is to do finitely many steps in a finite amount of time.

\footnotetext{
${ }^{12}$ This escape requires an extension of Kerr spacetime, this extension is shown in Figure 4.

${ }^{13} b^{-}$is the time $\mathrm{P}^{\prime} \mathrm{s}$ wristwatch shows when he falls through the outer event horizon.
} 


\section{$3 \quad$ Relativistic computers in a general setting}

Relativistic computers are not tied to rotating black holes, as there are other general relativistic phenomena on which they can be based. An example is anti-de-Sitter spacetime which attracts more and more attention in explaining recent discoveries in cosmology [19, 30]. Roughly, in anti-de-Sitter spacetime, time ticks faster and faster at farther away places in such a way that $\mathrm{P}$ can achieve infinite speed-up by sending away the computer $\mathrm{C}$ and waiting for a signal from her. This scenario is described and is utilized for computing non-Turing computable functions in [22]. This example shows that using black holes is not inherent to relativistic computers. It also shows that it is not necessary for the programmer to travel. Wormholes also can be used for constructing relativistic computers, see, e.g., [4]. In this latter scenario, neither the computer nor the programmer have to travel.

From a birds-eye view, the main parts of a relativistic computer as described in the previous section are a computer $\mathrm{C}$ (a Turing machine) with an infinite life for computing and a programmer $\mathrm{P}$ with two distinguished events in his life, one joint event $\mathrm{O}$ with the computer (when $\mathrm{P}$ programs $\mathrm{C}$ ) and another later event $\mathrm{E}$ when the programmer can evaluate the results of C's infinite life. This event $\mathrm{E}$ is such that $\mathrm{C}$ can send a signal arbitrarily late in her life such that it reaches $\mathrm{P}$ before E. Intuitively, the event from which $\mathrm{C}$ sends a signal which reaches $\mathrm{P}$ before E can be thought of as being "earlier" than E; and then one can think that E is "later" than any event in C's infinite life. If we think about it, it is surprising and hard to imagine at all that there can be an event "after" an infinite time has passed (on C's worldline). Such a thing cannot happen in our usual Euclidean space and "normal" time. General relativistic spacetimes with this property help our imagination, since they provide us with a consistent scenario in which this can happen. In this section, we define relativistic computers in abstract terms as consisting of a general relativistic spacetime together with two suitable worldlines. In the next section, we examine some realizability questions this setting raises.

A general relativistic spacetime is a structured set of events. An event is thought of as specified by which entities are present in it. We tend to call these entities (possible) observers, or bodies. The worldline of an observer is the set of all events in which this observer is present. The spacetime structure specifies which subsets of events are possible worldlines of observers and how time passes for these observers on their worldlines. This is done in the following manner. In a spacetime structure $\langle M, g\rangle$, the first component is 
a differentiable manifold (see, e.g., [45]). It specifies the large-scale topology of the spacetime, and it specifies which subsets of events count as "curves". In more detail, a differentiable manifold is a set $M$ of events together with a coherent system of one-to-one coordinate-functions (or charts) $\lambda_{i}: M_{i} \rightarrow R^{4}$ for $i \in I$, where $R^{4}$ is the set of all 4 -tuples of real numbers and $M$ is the union of the $M_{i}$ for $i \in I$. By "coherent" we mean that the worldview transformations $\lambda_{j} \circ \lambda_{i}^{-1}$ are smooth functions between open subsets of $R^{4}$. A "curve" is a parameterized subset of $M$ that is a smooth curve in all of the charts, or equivalently in some of the charts. The second component in a spacetime $\langle M, g\rangle$ is a metric tensor on $M$. This means that $g$ at each event specifies which curves passing through this event count locally as "travelable" by an observer and how time flows for this observer, and further which curves can be "traveled" by photons. These are called locally timelike and lightlike at the event, respectively. A curve is called timelike if it is locally timelike at each event in it, and similarly for lightlike. The worldlines in the spacetime are the timelike curves. Since the metric tensor $g$ specifies at each event how time locally passes, we can define the "timelike length", called proper time, along worldlines. This is what we called wristwatch time in section 2. A requirement for $g$ is that at each event the spacetime locally is like a special relativistic spacetime with a direction of time. A future-infinite worldline is a timelike curve which has infinite length to the future of any of its points. A curve is called causal if it is locally timelike or lightlike. It is thought that information can be sent along causal curves. The causal past of an event $\mathrm{E}$ is the set of all events $\mathrm{O}$ such that there is a causal curve with $\mathrm{O}$ and $\mathrm{E}$ on it such that $\mathrm{O}$ is earlier than $\mathrm{E}$ in the curve.

We are ready to define the notion of an abstract relativistic computer (Definition 3.1). For illustration of the definition below, see Figures 3(a) and 4.

Definition $3.1 A$ (n abstract) relativistic computer is a tuple $\left\langle M, g, \gamma_{C}, \gamma_{P}, O, E\right\rangle$ where $\langle M, g\rangle$ is a general relativistic spacetime, $\gamma_{C}$ is a future-infinite worldline with $O$ in it, $\gamma_{P}$ is a worldline with $O$ and $E$ in it such that $E$ is later than $O$ in $\gamma_{P}$ and $\gamma_{C}$ lies in the causal past of $E$.

A relativistic spacetime is called Malament-Hogarth $(\mathrm{MH})$ if it harbors a relativistic computer. This is equivalent to requiring that it contains an $\mathrm{MH}$ event, an event in whose causal past there is a future-infinite worldline. This definition appeared first in [21] and the name was coined in [13]; see also [12, sec.4.3]. 


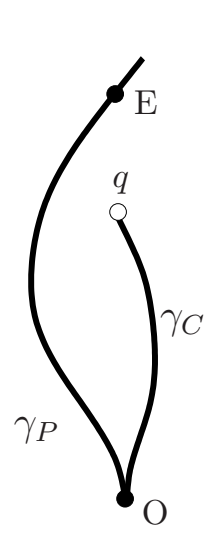

(a)

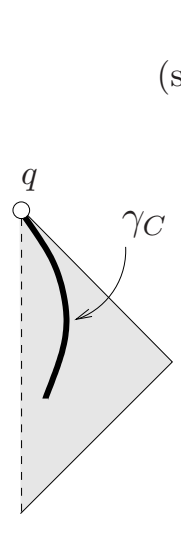

(b) future singularity this light signal (spacelike, unavoidable) does not reach $\gamma_{P}$

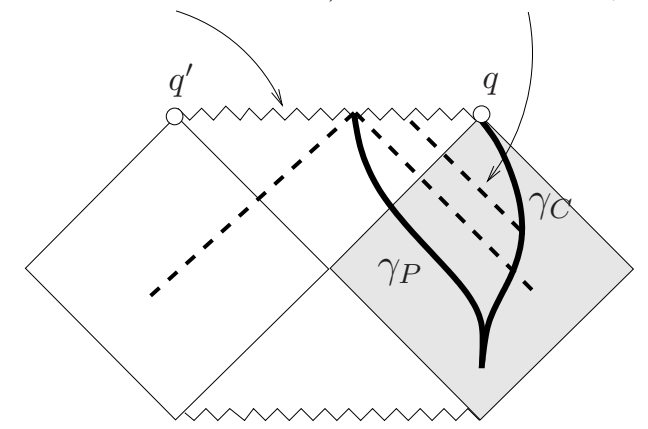

(c)

Figure 3: Schematic illustration of a relativistic computer as defined in Definition 3.1 is shown in (a). Conformal diagrams of Minkowski spacetime and Schwarzschild spacetime are shown in (b), and (c), respectively. It can be seen that these are not MH spacetimes. Lightlike direction is at 45 degrees, and directions which are more vertical than horizontal are timelike. Worldlines of infinite future length are those ending in a hollow circle.

Conformal, or Penrose, diagrams (see [11, sec.17]) of spacetimes are especially suitable for illustrating whether a spacetime is $\mathrm{MH}$ or not. Conformal diagrams are compactified 2-dimensional representations of spacetimes which illustrate causality relations faithfully, but distort metric length. Typically, they represent infinite regions with finite ones. Representing causality relations in conformal diagrams works as follows. Lightlike directions are at 45 degrees, the future direction is upward, and directions which are more vertical than horizontal are timelike. In our diagrams, there are hollow circles, and worldlines ending in these represent future-infinite ones.

Neither the Minkowski spacetime of special relativity nor the ordinary Schwarzschild black hole is MH: see Figure 3 (b) and (c). Therefore they do not support the existence of a relativistic computer. On the other hand, the spacetime of a rotating Kerr black hole is $\mathrm{MH}$ and any event within the inner event horizon (region III in Figures 1,2,4) is an $\mathrm{MH}$ event. In anti-de-Sitter spacetime each event is an MH event. The spacetime of an electrically charged black hole (called a Reissner-Nordström spacetime) is $\mathrm{MH}$, spacetimes with closed timelike curves are $\mathrm{MH}$, and there are many 
other examples [12, sec.4], [22, 26].

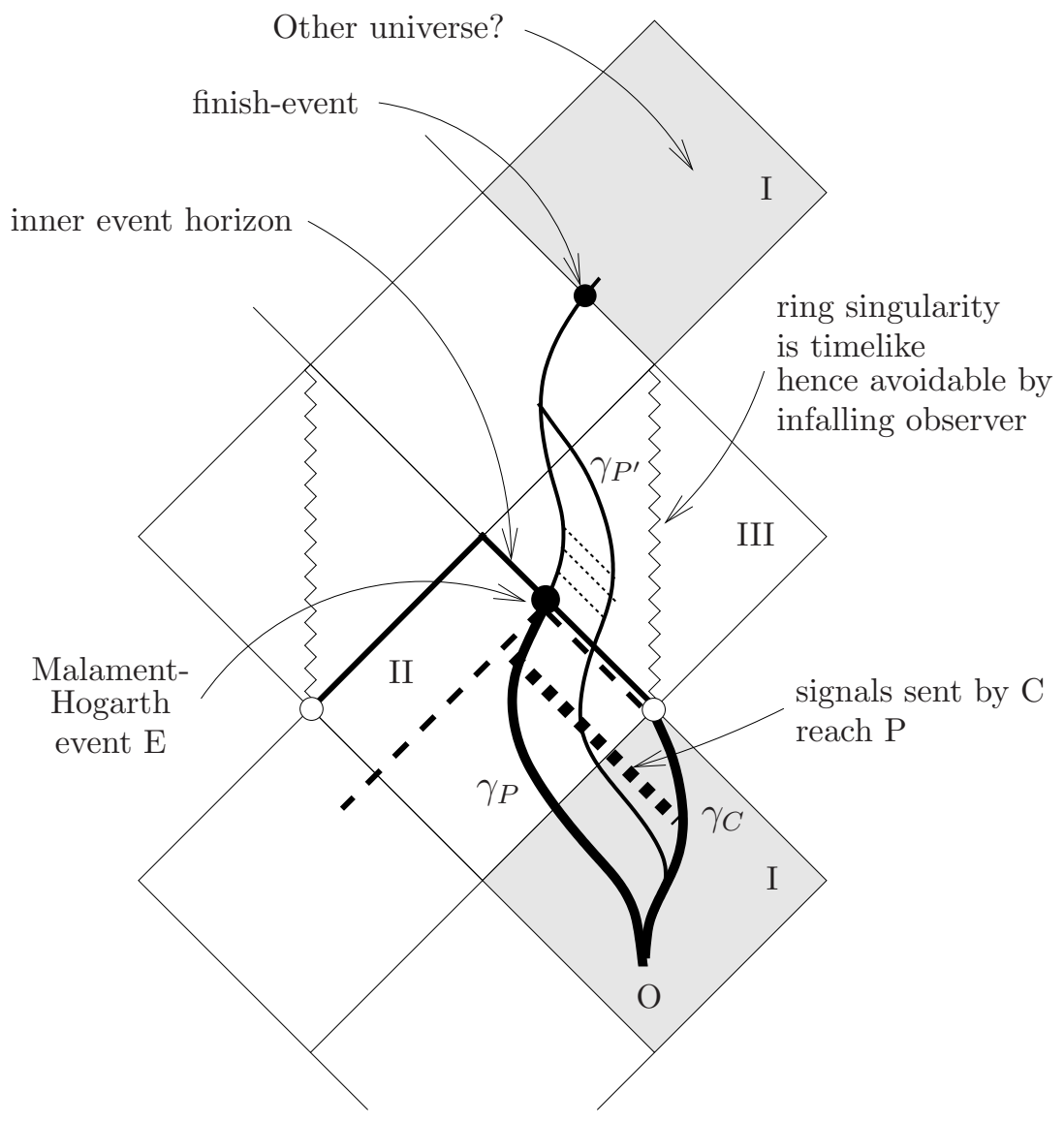

Figure 4: Conformal (or Penrose) diagram of a slowly rotating black hole. This is a Malament-Hogarth spacetime. The length of $\gamma_{P}$ is finite between $\mathrm{O}$ and $\mathrm{E}$, while the length of $\gamma_{C}$ is infinite and it lies in the causal past of E. So, $\left\langle\gamma_{C}, \gamma_{P}, O, E\right\rangle$ is a relativistic computer in this spacetime. We use $\gamma_{P^{\prime}}$ later, in section 4.1 .

As we said, an MH event is unusual: at this event some observer may see another observer disappearing from spacetime without a "visible cause". Let $\gamma: I \rightarrow M$ be a future-oriented timelike curve. We say that $p \in M$ is a future endpoint for $\gamma$ if $\gamma$ converges to $p$. When $\gamma$ is inextendible but it has a future endpoint $p$ not in its range, we can imagine that $\gamma$ "dies" at $p$ because its acceleration converges to infinity during its life. However, if $\gamma$ is 
finite and has no endpoint, then $\gamma$ is inextendible because "spacetime does not allow its extension". We can imagine that $\gamma$ "dies because spacetime does not provide the kind of event that $\gamma$ would need for continuing its life".

A nakedly singular event is defined as a spacetime point whose causal past contains a future-inextendible worldline without a future endpoint. (See [20, p.184], [22, pp.22,14,13], [12, Def.3.1 on p.74]. See also [18, 33].) By definition, then, each $\mathrm{MH}$ event counts as nakedly singular, since no futureinfinite $\gamma$ can have a future endpoint. ${ }^{14}$ The adjective "nakedly singular" sounds rather frightening, but what does it involve? Let us call an event strongly nakedly singular if we can see at that event worldlines of finite future length prematurely disappear (imagining that some horrible disruption of the spacetime called "singularity" killed the observers at that point of their lives). Our MH events are only "weakly" nakedly singular in that the worldlines that we see disappear are of infinite future length. We may see nothing unusual about an observer having this worldline. Why do we call MH events singular, then?

Singularities, or singular behavior, in the general relativistic spacetime literature are connected with break-down of predictability and determinism $[12$, p.66]. A function assigning real numbers to the events in a spacetime is called a global time. We say that a global time supports Laplacian determinism, roughly, when there is a time-instance such that (a) events with this time-tag form a reasonable "now", and (b) the arrangement of certain quantities computable in the spacetime at the events belonging to this "now" determines the whole spacetime. A requirement in (a) is that this "now" forms a spacelike hypersurface and the quantity in (b) is the Einstein-tensor. For a concrete definition, see e.g., [12, sec. 6.3]. This is a rather strong, global kind of determinism and spacetimes satisfying it are called globally hyperbolic. Hogarth [21] proved that no MH spacetime is globally hyperbolic. This is the theorem that justifies calling MH events "singular". ${ }^{15}$

\footnotetext{
${ }^{14}$ Here is a simple proof that arose in a correspondence with J. B. Manchak: Let $\gamma: I \rightarrow$ $M$ be a future-oriented future-infinite timelike curve and let $p \in M$ be arbitrary. There is a neighborhood $O$ of $p$ which does not contain infinite timelike curves. This follows from $g$ being locally special relativistic and from the continuity of $g$. Let $t \in I$ be arbitrary. Then $\gamma$ restricted to $\{s \in I: s \geq t\}$ is still a timelike curve of infinite length, thus $O$ cannot contain it. This shows that $\gamma$ does not converge to $p$.

${ }^{15}$ Penrose's strong Cosmic Censorship Hypothesis $(\mathrm{CCH})$ prohibits the existence of nakedly singular events. Etesi [15] describes many other interesting connections between variants of the $\mathrm{CCH}$ and relativistic computing.
} 
Thus, in order to have MH events, we have to give up existence of a global time supporting this strong determinism. However, assuming a weaker type of global time is consistent with $\mathrm{MH}$ spacetimes: see the definition of stable causality in the next section 4.1 .

How strange are MH spacetimes from a physical perspective? First, there are many MH spacetimes without strongly nakedly singular events, e.g., antide-Sitter spacetime. Here, as well as in Gödel's rotating universe, each event is weakly nakedly singular and there are no strongly nakedly singular events. Further, global hyperbolicity has not been generally accepted as a necessary condition for a spacetime to be physically realistic: see, e.g., [12, pp.97-99], $[45,27,16]$. Hogarth $[22$, p.83] emphasizes and explains that the presence of weakly nakedly singular events in and lack of global hyperbolicity of $\mathrm{MH}$ spacetimes does not render these spacetimes physically unrealistic. E.g., MH spacetimes do not have to be singular, which, following [20], means being timelike or lightlike geodesically incomplete. Hogarth emphasizes that there are nonsingular MH spacetimes in which carrying out the computation seems to be unproblematic. For example, anti-de-Sitter spacetime is such. Moreover, Manchak [28] shows that there is a clear sense in which general relativity allows for $\mathrm{MH}$ spacetimes to be brought about.

The above clears, we hope, several worries in the literature. E.g., [34] writes that $\mathrm{MH}$ spacetimes contain singularities and this might be an obstacle for relativistic computing. This worry is also quoted in [29]. As we outlined in the discussion above, it is mainly due to a matter of definition that $\mathrm{MH}$ events count as (weakly) nakedly singular: the breakdown of the kind of determinism they bring about (i.e., lack of global hyperbolicity) is not generally accepted as necessary for being physically realistic.

Is our own universe MH? Many of the MH spacetimes, like rotating black holes, may be built by a future, advanced civilization inside our usual "standard" universe according to high precision cosmology [19, ch 12]. Further, astronomers have observed objects that they think with great confidence are large rotating black holes [31, 1]. A typical example is the rotating 100million-solar mass black hole at the center of galaxy MCG-6-30-15.

\section{Physical realizability}

In this section, we briefly discuss some realizability/engineering questions in connection with relativistic computation. We concentrate on the plausibility 
of having reasonable communication between $\mathrm{C}$ and $\mathrm{P}$, and on the assumption of $\mathrm{C}$ having an infinite life.

\subsection{The blue-shift problem}

The blue-shift problem concerns communication between the computer and the programmer. Copeland [9] emphasizes the importance of this communication between $\mathrm{C}$ and $\mathrm{P}$, pointing out that an accelerating Turing machine as in section 2.1 cannot compute anything more than an unaccelerating one without a means to communicate with the "external world".

The blue-shift problem is a side-effect of our effort to make an infinite speed-up, but this side-effect can be cured with sufficient care. The essence of the problem is, that if $\mathrm{C}$ sends a message of, say, 100 successive signals in a time period of 1 second (C's time), the programmer $\mathrm{P}$ may receive these 100 signals in an arbitrarily small time-interval ( $\mathrm{P}$ 's time). By the same token, a light-signal (photon) sent by $\mathrm{C}$ can get arbitrarily blue-shifted when received by $\mathrm{P}$. Thus the energy of a photon sent by $\mathrm{C}$ can increase arbitrarily. This in turn might kill the programmer and also the programmer might not be able to decode the message.

For formulations of the blue-shift problem, see [12, p.111] and [26, p.279]. In these formulations, one assumes that the programmer and the computer communicate via light-signals: signals whose worldlines are lightlike geodesics. We say that we have a bounded blue-shift for an $\mathrm{MH}$ event together with the infinite worldline of $\mathrm{C}$ if there is an arrangement for $\mathrm{P}$ 's worldline together with a series of possible light-signals from a cofinal set of events of $\mathrm{C}$ to $\mathrm{P}$ such that the rate of frequency of a light-signal in this arrangement as observed by $\mathrm{C}$ and $\mathrm{P}$, respectively, is bounded. Otherwise, we say that we have a divergent blue-shift $[12,26]$.

A spacetime $\langle M, g\rangle$ is called stably causal ${ }^{16}$ if it possesses a global time function $t: M \rightarrow R$, where $R$ is the set of real numbers, which respects the flow of time on worldlines, i.e., whenever two events $p$ and $q$ are connected by a future-directed worldline such that $p$ is earlier than $q$ in this worldline, then $t(p)<t(q)$. Earman [12, Lemma 4.2 on p.111 and its converse in sec. 4.5] proves that, among the stably causal spacetimes, the MH ones are exactly those with a divergent blue-shift, if we assume the following hypothesis: The family of light-signals that $\mathrm{C}$ can send to $\mathrm{P}$ forms a 2-dimensional

\footnotetext{
${ }^{16}$ This is not the original definition, we use [12, p.166].
} 
integral submanifold in which the order of omission from $\mathrm{C}$ matches the order of reception at P. However, Manchak [26] constructs a stably causal MH spacetime with bounded blue-shift, and his construction exhibits many other desirable properties for a relativistic computer. He thus shows that in order to avoid the blue-shift problem we do not have to give up even the existence of a "good" global time-function, we only have to give up the above-cited hypothesis about a strong concert between the light-signals.

However, it is not necessary for $\mathrm{C}$ and $\mathrm{P}$ to communicate via lightsignals. ${ }^{17}$ Moreover, we do not need to stick with "geodesic messages". Several alternative means are offered in $\left[30\right.$, sec.5.3]. ${ }^{18}$ Here we recall one of those.

First of all, $\mathrm{C}$ does not send any photons towards $\mathrm{P}$. When she wants to send a message to $\mathrm{P}$, the computer $\mathrm{C}$ sends a postman $\mathrm{P}^{\prime}$ after $\mathrm{P}$ and asks $\mathrm{P}^{\prime}$ to carry her message to $\mathrm{P}$. This $\mathrm{P}^{\prime}$ is in a second spaceship chasing $\mathrm{P}$ and meeting $\mathrm{P}$ behind the inner event horizon sometime after the $\mathrm{MH}$ event but before an agreed upon time limit, e.g., before $\mathrm{P}$ re-enters region I at the "finish-event" as shown in Figure 4 where the worldline of postman $\mathrm{P}^{\prime}$ is also indicated. This $\mathrm{P}^{\prime}$ can be engineered in such a way that $\mathrm{P}^{\prime}$ catches up with $\mathrm{P}$ and hands the message to $\mathrm{P}$, e.g., in writing on paper (or some other material coding $)^{19}$. So, after the finish-event, $\mathrm{P}$ comfortably reads the message, say, the proof of the inconsistency of ZF and can decide whether or not it is correct. There are helpful "roadsigns" along this spacetime helping $\mathrm{P}$ to navigate. E.g., there are observable effects indicating that $\mathrm{P}$ has reached the finish-event, namely, the light of the "new universe" (the second appearance of region I) becomes visible for $\mathrm{P}$ when he reaches it. $\mathrm{P}$ does not have to wait for any information after the "finish-event". Summing up: P can decide whether ZF is consistent in bounded time (as we wanted). Namely, if ZF is inconsistent, a postman $\mathrm{P}^{\prime}$ will appear before the finish-event, hence $\mathrm{P}$

\footnotetext{
${ }^{17}$ Hogarth [22, p.87, last two paragraphs] already suggests communicating by other means than photons. He suggests to consider "switching the lights off" and "operate and communicate as purely mechanical devices (in a kind of billiard ball model)". Manchak [26, footnote 3] also hints at this possibility.

${ }^{18} \mathrm{~A}$ rather ingenious alternative way of communication is explored by Wüthrich [48]. He proposes that $\mathrm{C}$ keeps one of an entangled pair of particles while $\mathrm{P}$ takes the other with him.

${ }^{19}$ The details depend on whether there are limits of compressing information coded in a given amount of physical material. If there are no such limits then $\mathrm{P}^{\prime}$ carries the whole proof to $\mathrm{P}$. If there is such a limit, $\mathrm{P}^{\prime}$ carries only selected information about the existence and nature of the proof.
} 
learns about the inconsistency. (Even before this event, $\mathrm{P}$ will receive a light-signal soon after crossing the $\mathrm{MH}$ event from $\mathrm{P}^{\prime}$ indicating "you have mail, a message is coming to you".) If, on the other hand, ZF is consistent, $\mathrm{P}^{\prime}$ does not show up, and after hitting the finish-event, $\mathrm{P}$ will conclude that $\mathrm{ZF}$ is consistent.

An advantage of using a postman $\mathrm{P}^{\prime}$ for carrying the message to the programmer over using a photon or inertial body for carrying the message is the following. As opposed to the photon, $\mathrm{P}^{\prime}$ can navigate intelligently and can adapt to situations arising during his carrying of the message. Further, $\mathrm{P}^{\prime}$ can match velocities with $\mathrm{P}$ before sending the "you have mail" warning and before transferring the message itself.

A future task is to check whether $\mathrm{C}$ can send postman $\mathrm{P}^{\prime}$ after $\mathrm{P}$ with the message arbitrarily late in her life so that there is a common bound for the acceleration for these possible postmen. We believe that in the slow-Kerr spacetime this can be arranged.

\subsection{Infinity}

A worry about the present project might be that it requires unbounded time and space for the computer $\mathrm{C}$ to carry out her task. Luckily, presentday cosmological data (results of measurements) point in the direction that probably our universe is infinite both in time and space; see [19, Ch.11.10, 12.8]. For a similar conclusion, we also refer to [12, p.119] and [30, p.137]. Operating $\mathrm{C}$ also seems to require an unbounded amount of energy or matter. This worry is dealt with in detail in $[30,5.4 .3]$. Here we concentrate on one specific problem connected to infinity.

Piccinini $[35,34]$ finds it unlikely that any physical machinery can flawlessly operate for an infinitely long time. He says that the probability of error for such a machine is 1 . We think that the infinite jar with more and more balls example in $[29,1.3]$ shows that one has to be careful when generalizing from cases of unbounded finite time to infinite time. We briefly recall the example from [29]. Suppose we have a jar with the capacity to hold infinitely many balls. We also have a countably infinite pile of balls, numbered $1,2,3,4, \ldots$ First we drop balls $1-10$ into the jar, then remove ball 1 . Then we drop balls $11-20$ in the jar, and remove ball 2. Suppose that we continue in this way ad infinitum, and that we do so with ever-increasing speed, so that we will have used up our entire infinite pile of balls in finite time. How many balls will be in the jar when this task is over? One may answer: none. 
Namely, ball 1 was removed at the first stage, ball 2 was removed at the second stage, ball $n$ was removed at the $n$-th stage. Since each ball has a label $n$, each ball was removed at some stage, thus there will be no ball left in the jar at the end. This is so in spite that the number of balls in the jar tends to infinity as the number of stages approaches infinity. The case with probability 1 in Piccinini's argument is analogous to this example. The $\mathrm{MH}$ event is analogous to the end of the drawing process when there are no balls in the jar, and the error probability is analogous to the number of balls tending to infinity at every particular stage. Below we briefly describe a kind of management of time and space which seems to allow infinite operation with small error probability.

Analogously to the way life works on the Earth, instead of implementing the computer $\mathrm{C}$ by a single Turing machine, the programmer can use a fleet of self-reproducing robots which carry out the task of $\mathrm{C}$ in a massively parallel self-correcting fashion. Each robot lives for a finite time, and in each time instant there are finitely many robots. However, both numbers are unbounded, as our fleet of robots follow the strategy that sometime after each time, the computation is independently started from the beginning by more and more new robots with more and more life-expectancy. This kind of organization seems to ensure that if $\mathrm{P}$ did not receive a signal, then he would have confidence that there does not exist a proof of $x \neq x$ which the "society" of robots overlooked, e.g., because an accident killed all the robots. If one of the robots would find a proof of the inconsistency of ZF, this would not be sent to the programmer $\mathrm{P}$ automatically, but instead would be double-checked by the other robots and only in the case of a consensus would it be sent to $\mathrm{P} .{ }^{20}$

\section{Computational power}

In computability theory, there is a hierarchy for how hard it is to compute a task. We recall the basic notions from [7]. First, one codes the task to be about a set of numbers. For example, one gives an algorithm for coding the set-theoretic formulas as numbers, and then one considers the task of deciding whether the code of the formula $x \neq x$ is in the set of codes of theorems of ZF. After this, we deal only with sets of numbers.

\footnotetext{
${ }^{20}$ This kind of management of time and space with massive parallelism, redundancy and self-correcting processes like the way evolution of life on Earth works is presented in [30, 5.4].
} 
A set $S$ of natural numbers is called arithmetical if it can be defined by a formula of the first-order language having function symbols for zero, successor, addition and multiplication and a relation symbol for the ordering of natural numbers. This means that $S$ is called arithmetical when there is a formula $\varphi(x)$ of this language with one free variable $x$ such that $\varphi(n)$ is true in the structure of natural numbers if and only if $n \in S$. A similar definition holds for an $n$-place relation $S$ of natural numbers. An $n$-place relation is called $\Sigma_{1}$, or recursively enumerable, if it is definable by a formula of the form $\exists \bar{y} \varphi(\bar{y}, \bar{x})$, where $\varphi$ is a quantifier-free formula, $\bar{y}, \bar{x}$ are sequences of variables and the length of $\bar{x}$ is $n$. Continuing this way, assume that we have defined $\Sigma_{k}$ relations. A relation is called $\Pi_{k}$ if its complement relation is $\Sigma_{k}$, and a relation is called $\Delta_{k}$ if it is both $\Sigma_{k}$ and $\Pi_{k}$. We define a $\Sigma_{k+1}$ relation as one that can be defined by a formula of form $\exists \bar{y} \varphi(\bar{y}, \bar{x})$, where $\varphi(\bar{y}, \bar{x})$ defines a $\Pi_{k}$ relation. It can be proved that a relation is arithmetical if and only if it is $\Sigma_{k}$ or $\Pi_{k}$ for some $k$. A $\Delta_{1}$ relation is called decidable. Thus, a relation is decidable if and only if both it and its complement are recursively enumerable. It can be proved that a relation is decidable in the just defined sense if and only if there is a Turing machine that can decide about a sequence of numbers whether it is a member of the set, and a problem is recursively enumerable if there is a Turing machine that can enumerate the members of the set. There are recursively enumerable but not decidable sets, e.g., the set of all valid first-order formulas. A decidable task is called solvable or computable while all others are called non-computable or non-solvable.

The train of thought outlined in section 2 can be used to show that any recursively enumerable set can be decided by a relativistic computer [17]. Thus, relativistic computers can compute more than Turing machines can. The physical Church-Turing thesis is formulated in [10] as "whatever can be calculated by a machine (working on finite data in accordance with a finite program of instructions) is Turing-machine-computable." Since our relativistic computer operates on finite data and according a finite program, it challenges the physical Church-Turing thesis. Actually, relativistic computers can do more than deciding the recursively enumerable sets. Computability limits connected with relativistic computers are addressed by several authors [47]. For example, Welch [46, Theorem B] proves that the relations computable in Kerr black holes as presented in section 2.6 form a subclass of the $\Delta_{2}$ relations, and this is a proper subclass if and only if there is a fixed finite bound on the number of signals sent to the programmer on the finite length path. On the other hand, Hogarth $[22,23]$ proves that there are other $\mathrm{MH}$ 
spacetimes in which the members of the arithmetic hierarchy can be decided: for all $k$ he constructs a spacetime in which all $\Sigma_{k}$ relations can be decided by a relativistic computer.

\section{Conclusion}

We have used results of general relativity and cosmology for designing a relativistic computer that can compute a non-Turing computable function. We described, in the spacetime of a slowly rotating black hole, a scenario of a team consisting of an ordinary Turing machine computing for an infinite time and a programmer who can survey in finite time the result of this computation. We investigated how close this scenario can be brought to being physically realizable. A contribution of the present chapter is that we answer some concerns voiced in the literature.

Piccinini [34] closes his discussion of relativistic computing with two worries, namely that the error probability of an infinite computation is 1 , and that spacetimes supporting relativistic computers contain singularities. We answered the first concern in section 4.2, and we answered the second one in section 3. Another worry in the literature concerns communication between the computer and the programmer. The new-relative to [12, p.118], [26] or to [17] - feature we propose in section 4.1 is that we send the message not via light-signals but via a postman (not inertial and slower than the speed of light). It is a future task to check whether in the Kerr spacetime there is a sequence of worldlines for postmen starting from a cofinal set of events in the worldline of the computer such that there is a common bound for the acceleration in these worldlines.

\section{References}

[1] Abbott, B. P. et al., GW151226: Observation of gravitational waves from a 22-solar-mass binary black hole coalescence. Physical Review Letters $116,241103,2016$.

[2] Andraus, A., Zeno-machines and the metaphysics of time. Unisinos Journal of Philosophy 17,2 (2016), 161-167. 
[3] Andréka, H., Németi, I. and Németi, P., General relativistic hypercomputing and foundation of mathematics. Natural Computing 8,3 (2009), 499-516.

[4] Andréka, H., Németi, I. and Székely, G., Closed timelike curves in relativistic computation. Parallel Processing Letters 22,3 (2012), 1240010 (15 pages). World Scientific Publishing Company. DOI: $10.1142 / \mathrm{S} 0129626412400105$

[5] Bailey, J., Measurements of relativistic time dilatation for positive and negative muons in a circular orbit. Nature 268,5618 (1977), 301-305.

[6] Beggs, E., Costa, J. F., Tucker, J. V., Three forms of physical measurement and their computability. Review of Symbolic Logic 17,4 (2014), 618-646.

[7] Cooper, S. B., Computability theory. Chapman and Hall, 2004.

[8] Cooper, S. B., How can Nature Help Us Compute? In SOFSEM 2006: Theory and Practice of Computer Science - 32nd Conference on Current Trends in Theory and Practice of Computer Science, Merin, Czech Republic, January 2006 (Jiri Wiedermann, Julius Stuller, Gerard Tel, Jaroslav Pokorny, Maria Bielikova, editors), Springer Lecture Notes in Computer Science No. 3831, 2006, pp. 1-13.

[9] Copeland, J., Accelerating Turing Machines. Minds and Machines 12 (2002), 281-301.

[10] Copeland, J., The Church-Turing thesis. Stanford Encyclopedia of Philosophy, 2002. https: \\plato.stanford .edu \entries \church-turing \

[11] d'Inverno, R., Introducing Einstein's relativity. Clarendon Press, Oxford, 1992.

[12] Earman, J., Bangs, crunches, whimpers, and shrieks. Singularities and acausalities in relativistic spacetimes. Oxford University Press, Oxford, 1995.

[13] Earman, J. and Norton, J., Forever is a Day: Supertasks in Pitowsky and Malament-Hogarth Spacetimes, Philosophy of Science 60 (1993), 2242 . 
[14] Earman, J. and Norton, J., Infinite pains: the trouble with supertasks. In: S. Stich (ed.), Paul Benacerraf: the Philosopher and his critics. Blackwell, New York, 1994.

[15] Etesi, G., A proof of the Geroch-Horowitz-Penrose formulation of the strong cosmic censor conjecture motivated by computability theory. International Journal of Theoretical Physics 52,3 (2013), 946-960.

[16] Etesi, G., Exotica or the failure of the strong cosmic censorship in four dimensions. International Journal of Geometric Methods in Modern Physics 12,10 (2016), pp.1-11.

[17] Etesi, G. and Németi, I., Non-Turing computations via MalamentHogarth space-times. International Journal of Theoretical Physics, 41 (2002), 341-370.

[18] Geroch, R. and Horowitz, G. T., Global structure of spacetimes. In: General Relativity, Hawking, S. W., Israel, W. eds., Cambridge University Press, 1979. pp.212-293.

[19] Grøn, Ø. and Hervik, S., Einstein's general theory of relativity. With modern applications in cosmology. Springer, 2007.

[20] Hawking, S. W. and Ellis, G. F. R., The large scale structure of spacetime. Cambridge University Press, 1973.

[21] Hogarth, M., Does general relativity allow an observer to view an eternity in a finite time? Foundations of Physics Letters 5,2 (1992), 173-181.

[22] Hogarth, M., Predictability, computability, and spacetime. PhD Dissertation. University of Cambridge, 1996. 123pp.

[23] Hogarth. M., Deciding arithmetic using SAD computers. Brit. J. Phil. Sci. 55 (2004), 681-691

[24] van Leeuwen, J. and Wiedermann, J., The Turing Machine paradigm in contemporary computing. In B. Enquist, W. Schmid (eds.), Mathematics Unlimited - 2001 and Beyond. Springer-Verlag, Berlin, 2001. pp. 11391155. 
[25] Madarász, J. X., Németi, I. and Székely, G., First-order logic foundation of relativity theories. In: Mathematical Problems from Applied Logic II. New Logics for the XXIst Century. International Mathematical Series Vol 5, Springer, 2007. pp.217-252.

[26] Manchak, J., On the possibility of supertasks in general relativity. Foundations of Physics 40,3 (2010), 276-288.

[27] Manchak, J., What is a physically reasonable space-time? Philosophy of Science 78,3 (2011), 410-420.

[28] Manchak, J., Malament-Hogarth machines. Unpublished manuscript, 2017.

[29] Manchak, J. and Roberts, B. W., Supertasks. The Stanford Encyclopedia of Philosophy (Winter 2016 Edition), Edward N. Zalta (ed.). http://plato.stanford.edu/archives/win2016/entries/ spacetime-supertasks.

[30] Németi, I. and Dávid, Gy., Relativistic computers and the Turing barrier. Journal of Applied Mathematics and Computation 178 (2006), 118142.

[31] Niedzwiecki, A., Miyakawa, T., General relativistic models of the Xray spectral variability of MCG-6-30-15. Astronomy and Astrophysics 509 (2010), an. A22, 15pp.

[32] O'Neill, B., The geometry of Kerr black holes. A. K. Peters, 1995.

[33] Penrose, R., Singularities and time-asymmetry. In: General Relativity, Hawking, S. W., Israel, W. eds., Cambridge University Press, 1979. pp.581-638.

[34] Piccinini, G., The physical Church-Turing Thesis: modest or bold? British Journal for the Philosophy of Science 62,4 (2011), 733-769.

[35] Piccinini, G., Computation in physical systems. Stanford Encyclopedia of Philosophy (Jul. 21, 2010). E. N. Zalta (ed.) Center for the Study of Language and Information, Stanford University, Stanford. http://plato.stanford.edu 
[36] Pitowsky, I., The physical Church Thesis and physical computational complexity. Iyyun 39 (1990), 81-99.

[37] Pogge, R. W., Real-world relativity: the GPS navigation system. March 2017. http://www . astronomy .ohio-state.edu/ pogge/Ast162/Unit5/ gps.html

[38] Rindler, W., Relativity. Special, general and cosmological. Oxford University Press, 2001.

[39] Shagrir, O. and Pitowsky, I., Physical hypercomputation and the Church-Turing Thesis. Minds and Machines 13 (2003), 87-101.

[40] Syropoulos, A., Hypercomputation (computing beyond the ChurchTuring barrier). Springer, 2008. xiii+243 old.

[41] Stannett, M., The computational status of physics: a computable formulation of quantum theory. Natural Computing 8,3 (2009), 517-538.

[42] Taylor, E. F. and Wheeler, J. A., Black holes. Addison, Wesley, Longman, San Francisco, 2000.

[43] Thorne, K. S., Black holes and time warps. Einstein's outrageous legacy. W.W. Norton and Co., 1994.

[44] Tipler, F. J., The physics of immortality. Anchor Books, New York, 1994.

[45] Wald, R., General relativity. University of Chicago Press, 1984.

[46] Welch, P. D., The extent of computation in Malament-Hogarth spacetimes. The British Journal for the Philosophy of Science 59,4 (2008), 659674 .

[47] Wiedermann, J. and van Leeuwen, J., Relativistic computers and nonuniform complexity theory. In: Calude et al (eds.) UMC 2002. Lecture Notes in Computer Science Vol. 2509, Springer-Verlag, Berlin, 2002. pp.287-299.

[48] Wüthrich, C., A quantum-information-theoretic complement to a general-relativistic implementation of a beyond-Turing computer. Synthese 192,7 (2015), 1989-2008. 
Alfréd Rényi Institute of Mathematics, Hungarian Academy of Sciences Budapest, Reáltanoda st. 13-15, H-1053 Hungary 